\title{
Research on Magnetic Valve Structure Optimization of Magnetic Controlled Reactor
}

\author{
Xiangzheng $\mathrm{Xu}^{*}$
}

Department of Electronics Engineering, East China Jiao-Tong University, Nanchang, 330013, China

\begin{abstract}
This paper analyzes the power loss of magnetically controlled reactor based on the basic theory of the electromagnetic field. In a variety of magnetic valve structure models as the object, physical simulation models of the reactor are built. Using Ansoft Maxwell simulation software, various magnetic valve structures of the reactor are studied by the analysis of the magnetic valve type reactor core and coil power loss. The simulation results show that the reactor's magnetic field distribution of the multistage magnetic valve structure is uniform, and inhibition effect of edge effect is obvious. The reactor power loss of multistage magnetic valve structure is least. Core loss is $47.6 \%$ lower than a single stage magnetic valve reactor, and coil eddy current loss is $62.9 \%$ lower than that of single stage magnetic valve reactor.
\end{abstract}

Keywords: Magnetic valve structure, Magnetically controlled reactor, Power system, Eddy current losses, MCR.

\section{INTRODUCTION}

Magnetically controlled reactor (MCR) is an important device for voltage control and reactive power compensation of power systems. In recent years, considerable attention paid to it has gradually widened the scope of its application. Magnetically controlled reactor works by means of controlled current control loop of DC excitation to change the magnetic saturation of the core, to achieve smooth adjustment reactive power output. It has obvious advantages for a wide range of voltages, high reliability, less occupied area and maintenance convenience. It is a static and a good application prospect of reactive power compensation device [1]. In order to cut the loss and harmonic content, usually magnetic valve reactor is taken. Single stage magnetic valve reactor can decrease the amount of harmonics. According to the relevant literature, due to the magnetic valve alternate saturation, in the region near the magnetic valve, there exists a radial component of the magnetic field which adds additional loss of the reactor core and winding [2]. So it is necessary to improve the structure of existing magnetic valve.

Many scholars and research institutions have conducted in-depth research on the structure of magnetically controlled reactor magnetic valve and have made a number of achievements. The physical simulation modes of magnetically controlled reactor are established by using Ansoft Maxwell simulation software and the reasonable magnetic valve structure for magnetically controlled reactor are explored, in order to suppress edge effects, and effectively reduce the power loss of the core and winding of magnetically controlled reactors.

*Address correspondence to this author at the Department of Electronics Engineering, East China Jiao-tong University, Nanchang, 330013, China; Tel: 13970977065; E-mail: ecjtuxxz@163.com

\section{PRINCIPLE OF MCR}

Fig. (1) is single-stage magnetic valve type MCR diagram. As shown in Fig. (1), the main core of magnetically controlled reactor is split into two parts, namely core I and core II, cross-sectional area is $A_{b}$ and length is $l-l_{t}$. Small cross sectional height is equal to $l_{t}$, its width is $b$, and cross-sectional area is $A_{b t}$. Four coils with numbers of turns, $\mathrm{N} / 2$, are wound symmetrically around two half core columns. There are two taps with the tap ratio of $\delta$ on the winding of every half iron core column. There are two thyristors, $T_{1}$ and $T_{2}$, between taps. Different core windings up and down are in parallel to the grid power supply after cross connection. The fly-wheel diode is across the cross section on the endpoint. Throughout the regulating range of the capacity, only a small area of magnetic circuit is saturated and the rest is in an unsaturated state. The capacity of the reactor can be changed by changing the magnetic saturation degree of the small section [3].

\section{ANALYSIS OF THE POWER LOSS FOR MCR}

\subsection{The Core Losses of MCR}

Because of the presence of the alternating or pulsed magnetic field in the reactor core, it often causes the loss of power. The loss is in the form of heat. Power loss also includes hysteresis losses and eddy current losses. Numerous factors affect the power loss of MCR. The power loss can be summarized by Eq. 1 [4]:

$P=k_{n} B_{\max }^{2} f+k_{c}\left(B_{\max } f\right)^{2}+k_{e}\left(B_{\max } f\right)^{1.5}$

Among them, $k_{n}$ is hysteresis coefficient.

$k_{c}$ is eddy coefficient. 
$B_{z}$ is additional eddy coefficient that is formed due to magnetic domain of the ferromagnetic layer.

$f$ is the voltage frequency applied to the reactor.

$B_{\max }$ is the maximum magnetic flux density of the core $(\mathrm{W} / \mathrm{m} 2)$.

According to transformer theory, the induction potential of primary side is supposed to be $E_{1}$ which is determined by Eq. 2:

$$
E_{1}=k f B_{\max }
$$

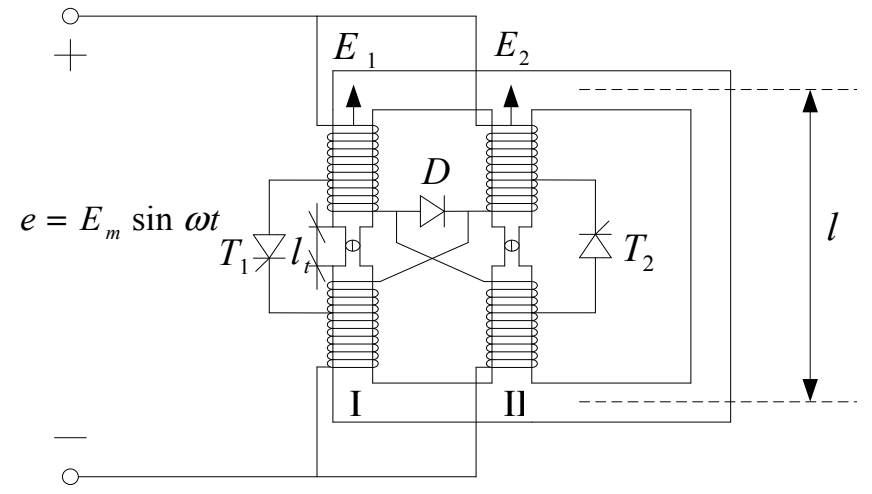

Fig. (1). Principle diagram of magnetic valve type MCR.

Among them, $\mathrm{k}$ is proportional constant. And it is determined by the primary side turns and the sectional area of the iron core. The power loss is shown in [5]:

$P=k_{n}\left(\frac{E_{1}}{k}\right)^{2} \frac{1}{f}+k_{c}\left(\frac{E_{1}}{k}\right)^{2}+k_{e}\left(\frac{E_{1}}{k}\right)^{1.5}$

Owing to the primary side impedance voltage drop of the reactor as being very small, if it is ignored, the $E_{1}=U_{1}$. This demonstrates that the loss of reactor and the applied voltage has very important relations. If $U_{1}$ is a certain value, then the loss of the reactor core is constant. If the voltage is wavy, then the loss of reactor is changed. Reactor core loss is related to core material, magnetic valve structure as well as the core manufacturing process and so on, but it is not concerned with the size of the load [6].

\subsection{Eddy Current Losses of the Reactor Coil}

In fact, the eddy current loss of magnetic control reactor coil is caused by the leakage magnetic field of the magnetic control reactor. In general, the greater the capacity of magnetic control reactor, the stronger the magnetic field leakage, causing the eddy current loss to increase. The increase of the eddy current losses causes internal overheating of the magnetic control reactor, and its thermal performance becomes poor, eventually it leads to the insulation aging and early breakdown [7].

The law of electromagnetic induction states that the electromotive force of winding leakage reactance is in proportion to the winding leakage flux. Therefore, leakage reactance electromotive force of the winding is proportionate to the load current and the turns of the windings, and determined by the winding geometry. Magnetic flux leakage of windings includes longitudinal and transverse magnetic flux leakage. Longitudinal leakage magnetic flux is parallel in axial direction of winding. It produces corresponding longitudinal leakage reactance potential. Transverse magnetic flux leakage and the vertical magnetic flux leakage with axial direction of windings induce transverse leakage reactance potential collectively.

The greater the capacity of magnetic control reactor, the greater the eddy current loss caused by transverse magnetic flux leakage [8]. The loss distribution of the coil caused by the transverse component of magnetic leakage is very uneven. In general, both ends of magnetically controlled reactor winding produce the biggest transverse magnetic flux leakage and achieve the highest temperature. Therefore, ends of the reactor winding are dangerous areas of local overheating.

The vertical eddy current loss is due to the vertical magnetic flux leakage. The size of it is related to the wire size [9]. According to the magnetic flux density and distribution characteristics of the winding, the values of a finite unit magnetic flux density can be given by (4):

$B=B_{r} r+B_{z} z$

Among them, $B_{r}$ is the unit transverse flux density, and $B_{z}$ is the perpendicular magnetic flux density.

Winding eddy loss is calculated by the $\mathrm{B}$ of finite element unit. The eddy current loss calculation formula of the unit volume can be expressed as follows [10]:

$P_{E}=\frac{1}{24 \rho} \omega^{2} d^{2} B^{2} \quad W / m^{3}$

If the subdivision unit is a triangle, then along the circumference of each unit, eddy current loss in the volume can be achieved.

The transverse eddy current loss is given as follows:

$P_{E r i}=\frac{1}{24 \rho}\left(B_{r i} b \omega\right)^{2} 2 \pi R_{i} S_{i}$

The vertical eddy current loss is expressed as follows:

$P_{E z i}=\frac{1}{24 \rho}\left(B_{z i} d \omega\right)^{2} 2 \pi R_{i} S_{i}$

So, the total eddy current loss of a unit is gained:

$P_{E i}=P_{E r i}+P_{E z i}$

Among them, $B_{z i}$ is the vertical magnetic flux density of $I^{\text {th }}$ units, and $B_{r i}$ is transverse magnetic flux density of $I^{\text {th }}$ unit. $\mathrm{b}$ and $\mathrm{d}$ are the unit vertical height and unit crosswise width, respectively, $\omega$ is the angular frequency, $\rho$ is winding resistivity, $\mathrm{Si}$ is the area of $I^{\text {th }}$ unit conductor, $R_{i}$ is distance between the $I^{\text {th }}$ unit center of 
gravity and the core center. Eddy current loss of the windings can be expressed as follows:

$$
P_{E}=\sum_{i=1}^{N}\left(P_{E r i}+P_{E z i}\right)
$$

\section{THE MAGNETIC VALVE OPTIMIZATION ANALYSIS OF MCR CONFLICT OF INTEREST}

Taking multi-stage magnetic valve structure model of the Fig. (2) for example, and taking the geometric center of reactor for the reference coordinate system origin, according to the symmetry of core structure, a layer of iron core lamination acts as object of magnetic field analysis, and to solve the field model it is established as shown in Fig. (3).
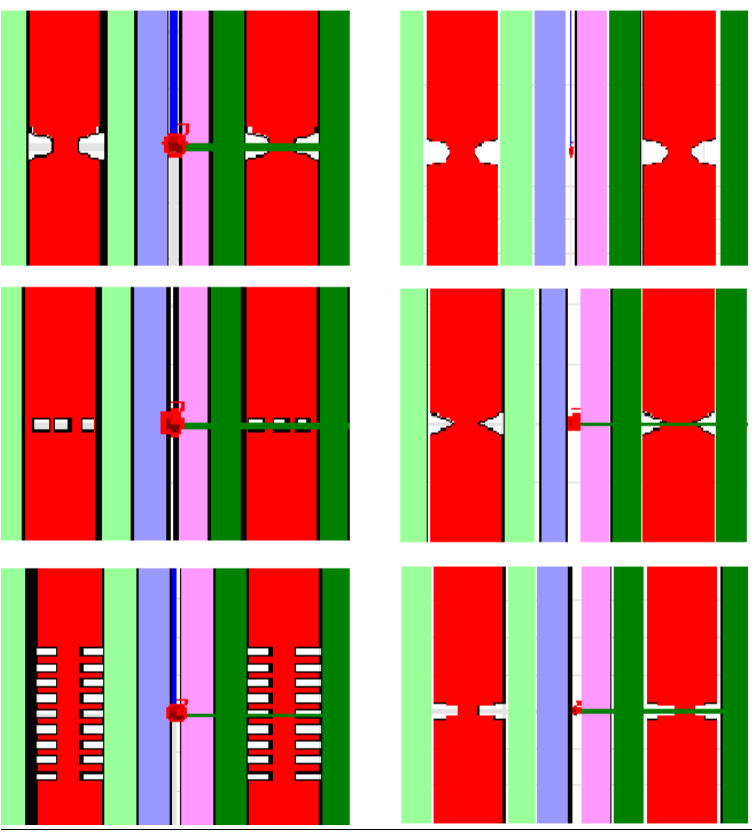

Fig. (2). Magnetic valve structure model.

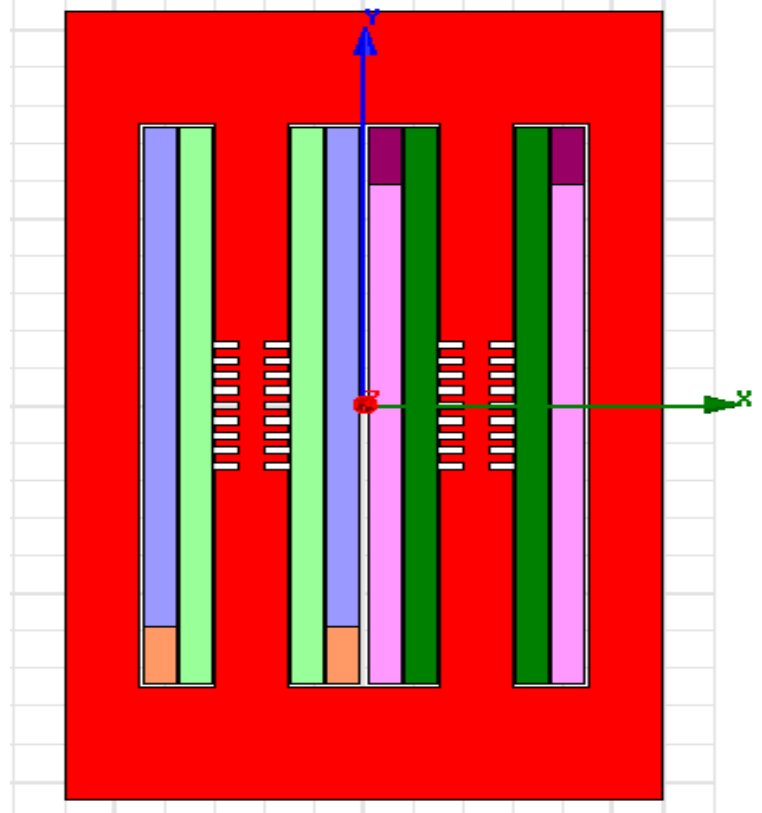

Fig. (3). Solving field model of MCR.
Assuming that the type of core is silicon steel, $k_{n}$ $=201.758, k_{c}=0.726$, $k e=0, \mu_{n}=2000000 \mathrm{~s} / \mathrm{m}$, weight density $\sigma=7872 \mathrm{~kg} / \mathrm{m} 3$, model thickness $\delta=0.06 \mathrm{~m}$. At the same time, it is assumed that the relative permeability of coil $\mu_{r}=0.99996, \mu_{n}=58000000 \mathrm{~s} / \mathrm{m}$, weight density $\sigma$ $=8933 \mathrm{~kg} / \mathrm{m} 3$, air relative permeability $\mu_{r}=1$. The coils have an appropriate value current, and the reactor is in a semi-limit saturated state. This paper optimizes the structure of the magnetic valve through Ansoft Maxwell software. The field distributions of single-stage magnetic control reactor are shown in Figs. (4-9). The field distributions of multi-stage magnetic control reactor are shown in Figs. (10-15).

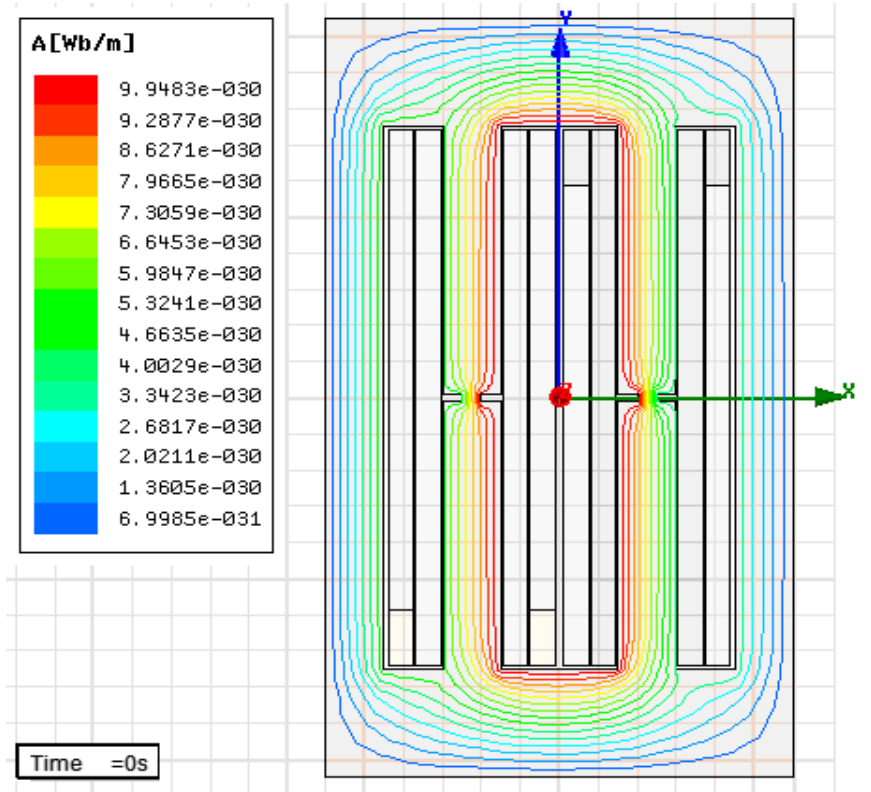

Fig. (4). Magnetic field distribution of single-stage. MCR when the time is $0 \mathrm{~s}$.

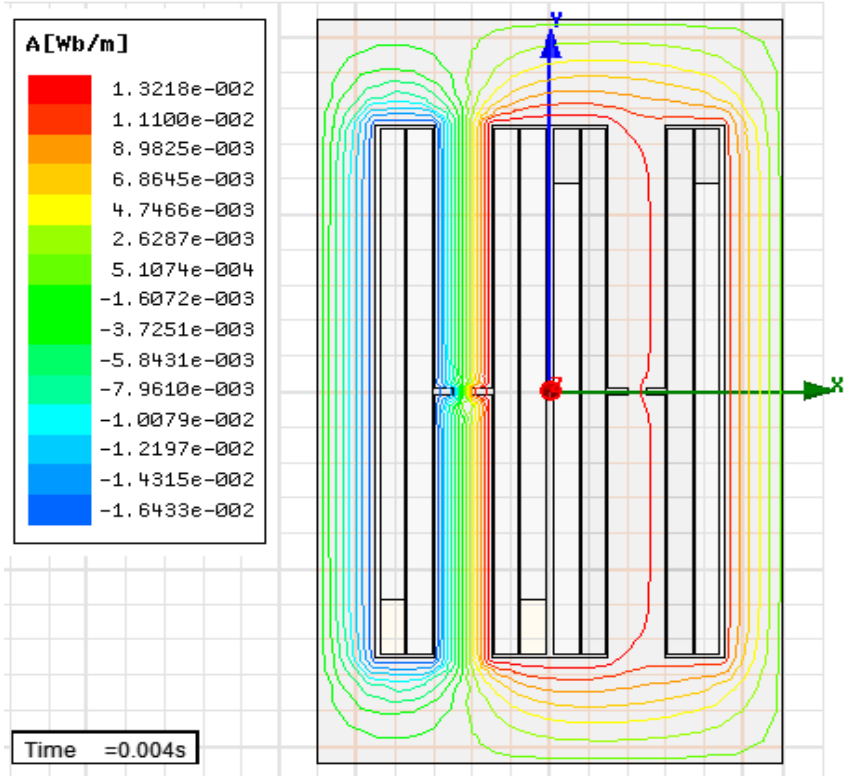

Fig. (5). Magnetic field distribution of single-stage. MCR when the time is $0.004 \mathrm{~s}$. 


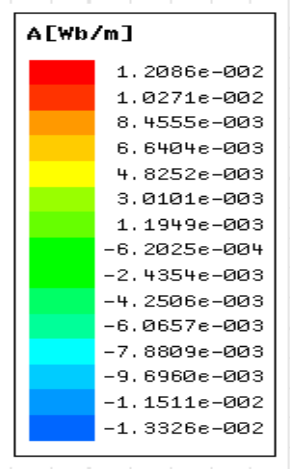

Time $=0.008 \mathrm{~s}$

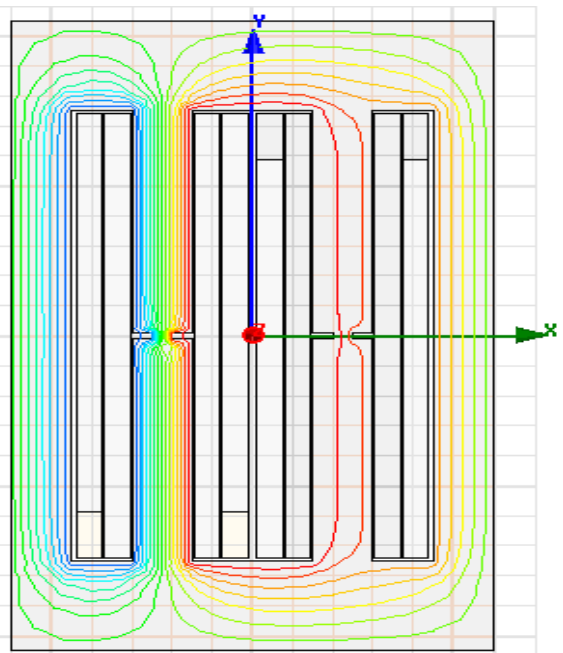

Fig. (6). Magnetic field distribution of single-stage. MCR when the time is $0.008 \mathrm{~s}$.

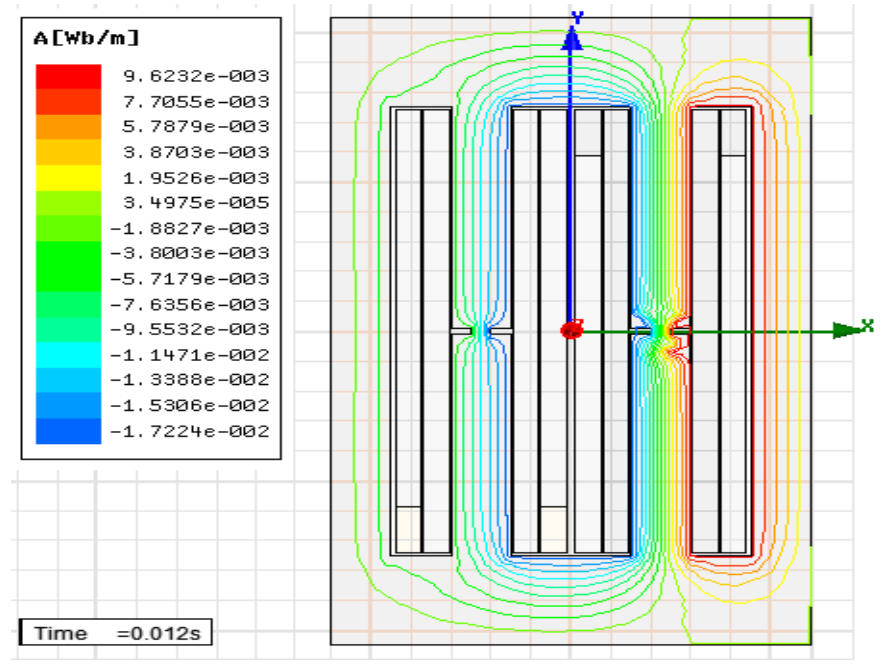

Fig. (7). Magnetic field distribution of single-stage. MCR when the time is $0.012 \mathrm{~s}$.

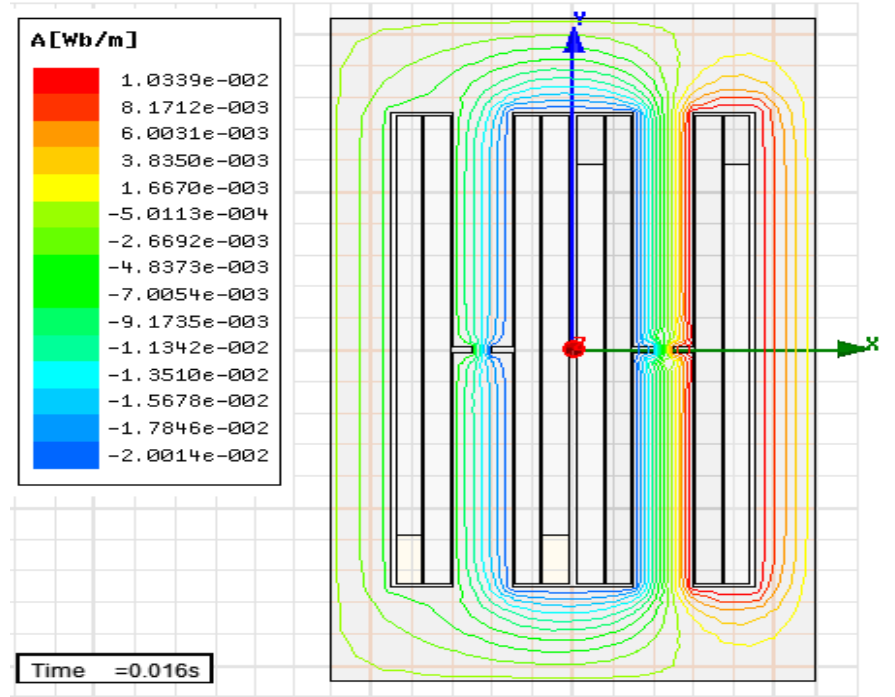

Fig. (8). Magnetic field distribution of single-stage. MCR when the time is $0.0016 \mathrm{~s}$.

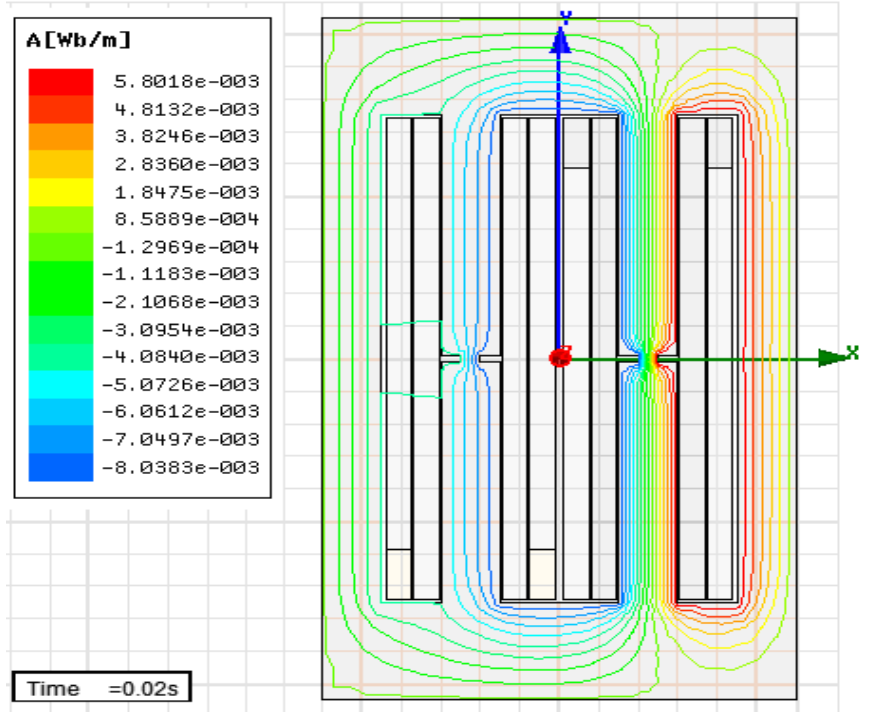

Fig. (9). Magnetic field distribution of single-stage. MCR when the time is $0.02 \mathrm{~s}$.

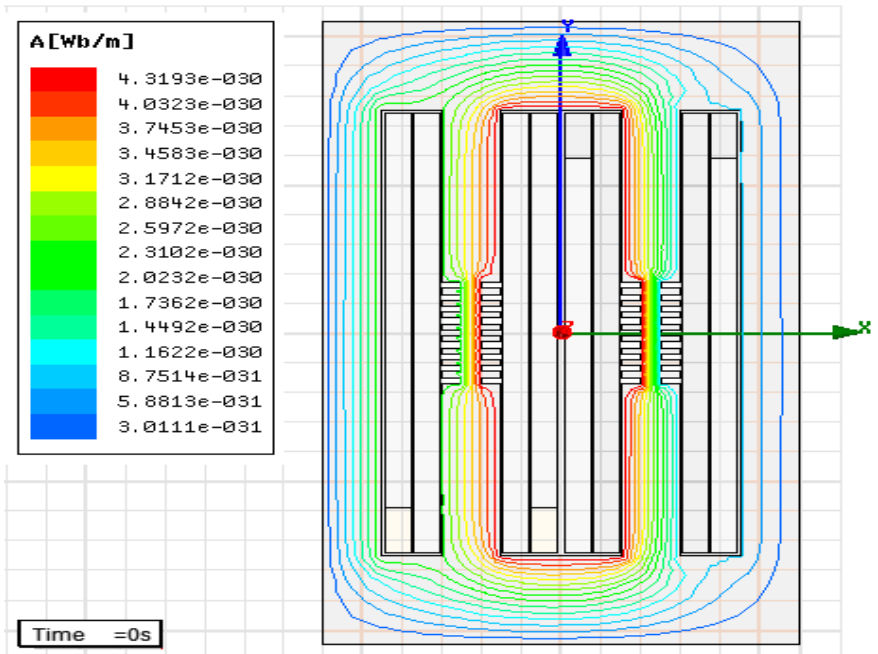

Fig. (10). Magnetic field distribution of multi-stage. MCR when the time is $0 \mathrm{~s}$.

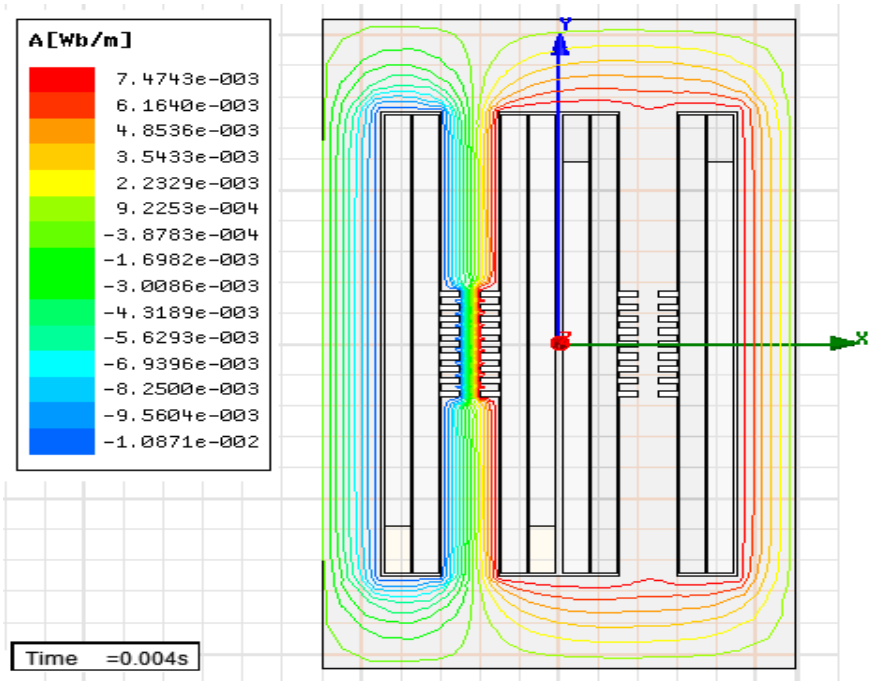

Fig. (11). Magnetic field distribution of multi-stage. MCR when the time is $0.004 \mathrm{~s}$. 


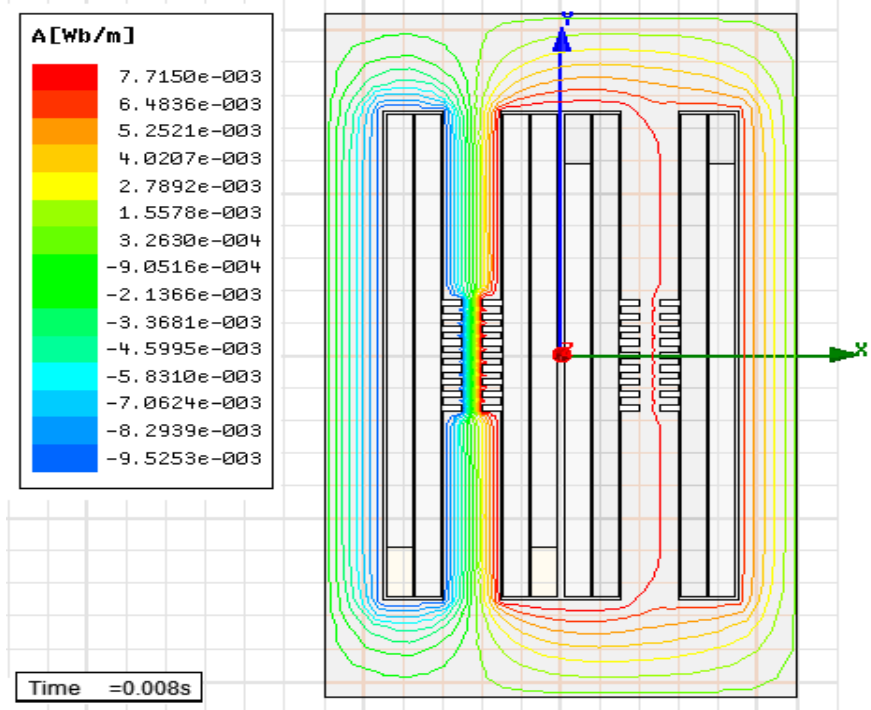

Fig. (12). Magnetic field distribution of multi-stage. MCR when the time is $0.008 \mathrm{~s}$.

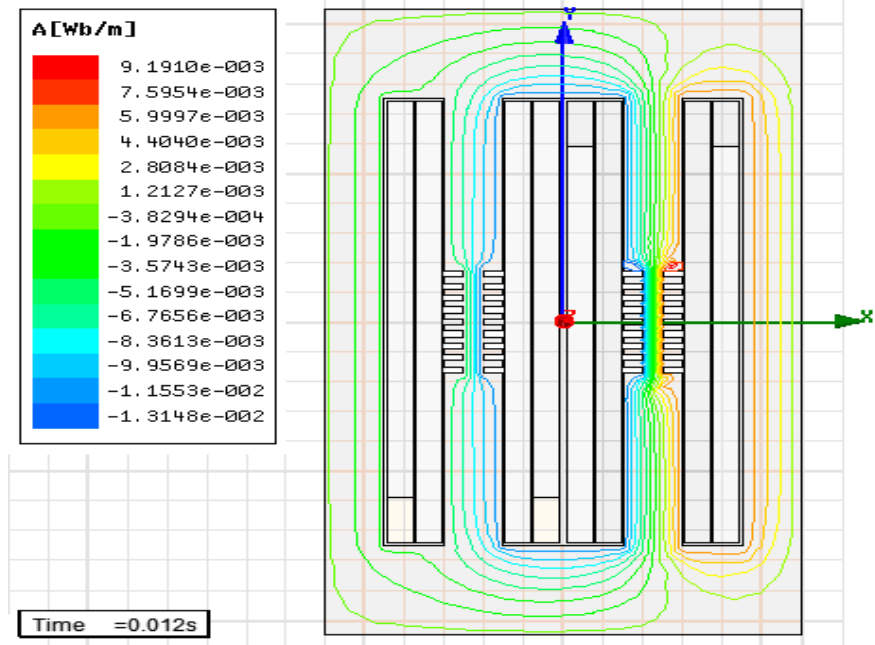

Fig. (13). Magnetic field distribution of multi-stage. MCR when the time is $0.012 \mathrm{~s}$.
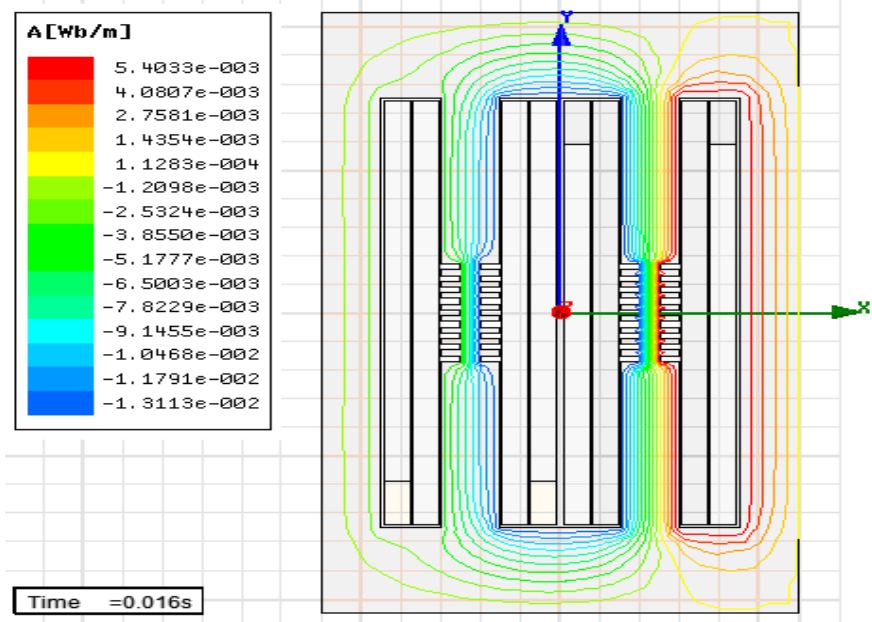

Fig. (14). Magnetic field distribution of multi-stage. MCR when the time is $0.016 \mathrm{~s}$.

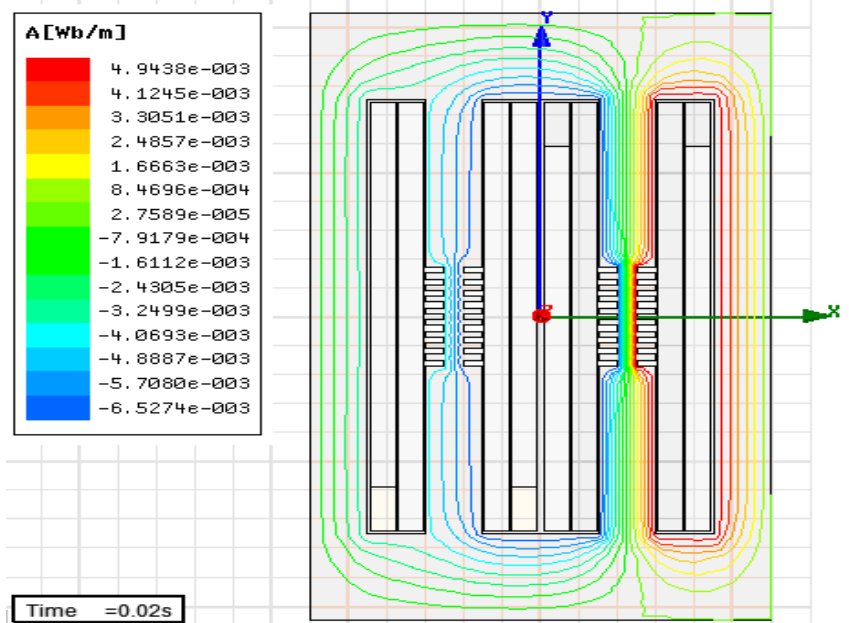

Fig. (15). Magnetic field distribution of multi-stage. MCR when the time is $0.02 \mathrm{~s}$.

Figures above show the magnetic field distribution of the iron core in a semi-limited saturated state. When current is in the positive half cycle, left iron core is in the saturated state. But, when the current is in the negative half cycle, the right side of the core is in the saturated state. In a cycle, both side cores carry current in turn. As can be seen from Figs. (4-9), the edge of the effect is obvious around the magnetic valve. And because of the deviation of magnetization curve, local irregular curve of magnetic field distribution is produced in the core material. A portion of the magnetic flux is perpendicular to the silicon steel sheet. It produces the large eddy current loss and forms local overheating. Irregular curved magnetic flux further increases the eddy current loss, and accelerates the process of the magnetic control reactor aging. At the same time, part of the magnetic flux also enters winding area, and forms magnetic flux leakage, and a large eddy current loss is produced in the coil. As can be seen from Figs. (10-15), the magnetic field lines are ruled and smoothed for multi-stage magnetic valve MCR, and its inhibiting effect of edge effect is obvious. Two types of power loss curve are shown in Figs. $(\mathbf{1 6}, \mathbf{1 7})$ respectively.

As can be seen from the Figs. $(\mathbf{1 6}, \mathbf{1 7})$, the core power loss of magnetic valve type reactor is usually the greatest when the current is at the largest gradient. Coil windings of magnetic valve type reactor have larger power loss during the current increase. Due to MCR of multistage magnetic valve structure, the edge effect is restrained. It greatly reduces the loss of the core and coil, controls the MCR internal local overheating, and improves the thermal stability of the insulating material. The results of the power loss optimization analysis are as shown in Table $\mathbf{1}$.

The simulation results of Table $\mathbf{1}$ show that the multistage magnetic valve reactor is effective to suppress the edge effects. The core loss of reactor was $47.6 \%$ lower than a single stage magnetic valve type reactor core. The eddy current loss caused by the edge effect was reduced to $62.9 \%$, lower than MCR of a single-stage magnetic valve. 


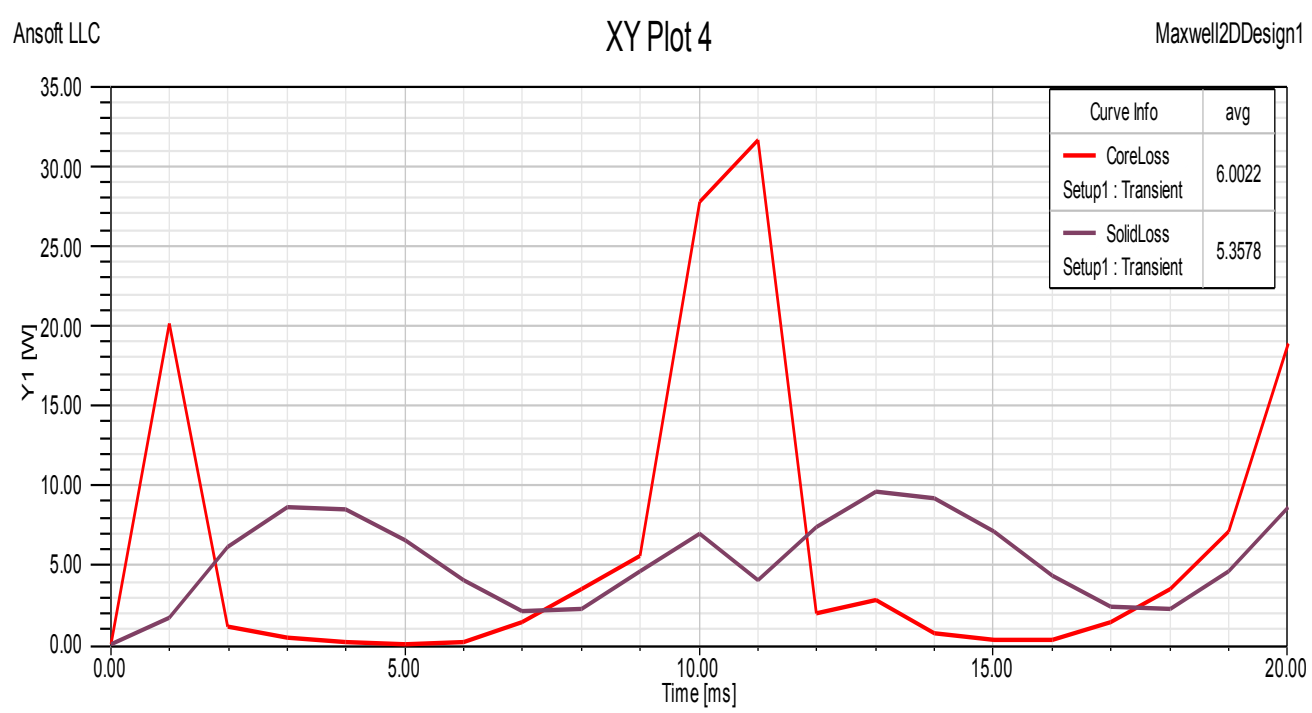

Fig. (16). Power loss of single-stage MCR.

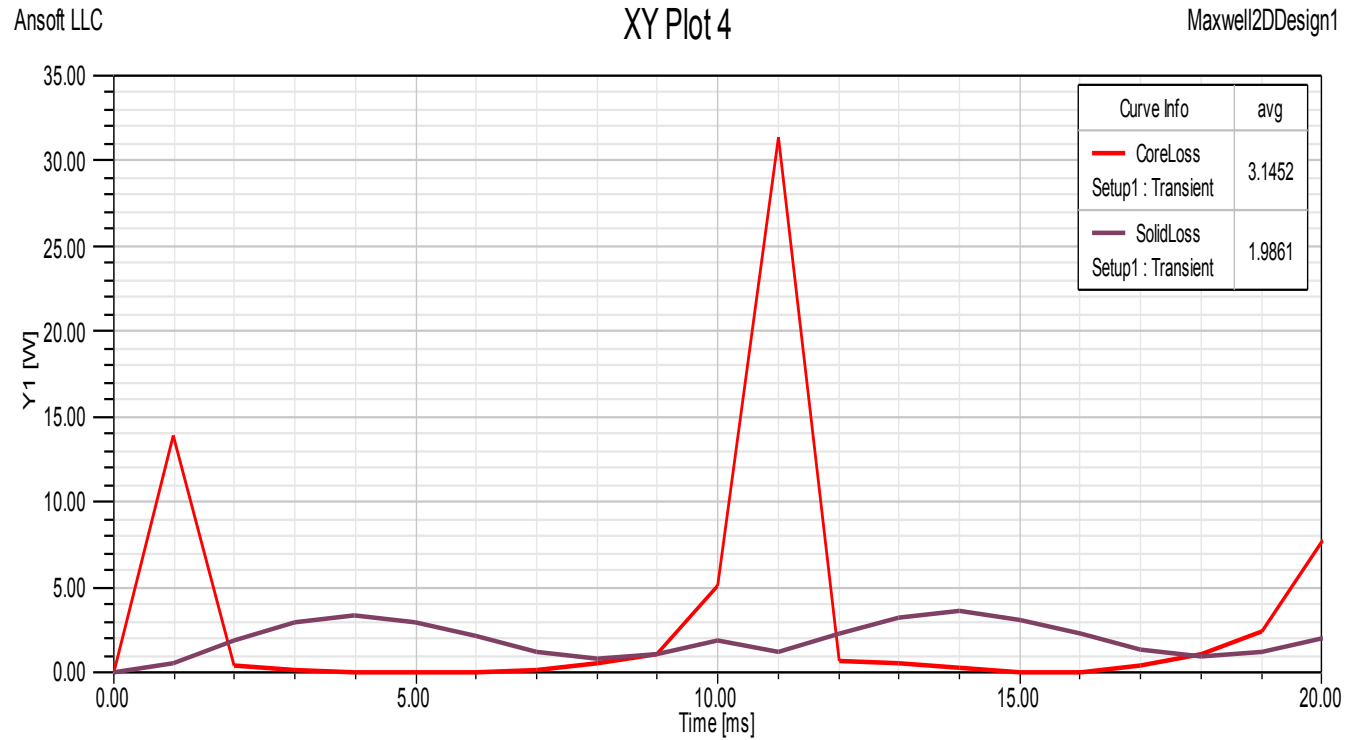

Fig. (17). Power loss of multi-stage MCR.

Table 1. Power loss value of core and windings.

\begin{tabular}{|c|c|c|}
\hline & Coil Loss $(\mathbf{W})$ & Winding Loss $(\mathbf{W})$ \\
\hline \hline Trapezoid & 5.3494 & 4.8831 \\
\hline Elliptic type & 6.9368 & 5.1073 \\
\hline Triangle & 10.4743 & 3.3581 \\
\hline Inner hole & 8.1778 & 4.8599 \\
\hline Multistage type & 3.1452 & 1.9861 \\
\hline Single stage type & 6.0022 & 5.3578 \\
\hline Step type & 6.0393 & 4.8955 \\
\hline
\end{tabular}

\section{CONCLUSION}

The magnetically controlled reactor has obvious advantages, especially high reliability and good economy. Therefore, it has received extensive attention in recent years and its extensive application has been increased gradually. The simulation analysis of the magnetic valve structure reveals that the magnetic field distribution is uniform in MCR of multistage magnetic valve structure, and lines of magnetic force are regular and smooth. Edge effect suppression effect is palpable. Therefore, multistage magnetic valve reactor power loss is the smallest, the core loss is $47.6 \%$ lower than the single stage magnetic valve type, and coil eddy current losses are $62.9 \%$ lower than that of a single stage magnetic valve reactor.

\section{CONFLICT OF INTEREST}

The author confirms that this article content has no conflict of interest.

\section{ACKNOWLEDGEMENTS}

This paper is supported by Jiangxi Province Department of Science and Technology under grant No.20122BAB206028. 


\section{REFERENCES}

[1] C. Baichao, New Controllable Saturated Reactor Theory and Application. Wuhan School of Water Resources and Hydropower Wuhan, Engineering Press, 1999.

[2] L. Wenbiao, C. Hanxiang, and W. Bin, "Study on the harmonic characteristics of the magnetic valve controllable reactor based on classification," Guangdong Electric Power, vol. 26, pp. 9-13, 2012.

[3] Z. Shishuo, Y. Zhongdong, and W. Xuan, "Model and experimental study of mer simulation," Electrical Applications, vol. 32, pp. 52-55, 2013.

[4] Z. Weijie, and Z. Xiaoxin, "Magnetically controlled shunt reactor equivalent reactance transient model based on dynamic reluctance", In: Proceedings of the CSEE, 2011, pp. 1-6.

[5] Z. Jianhua, and H. Zhao, "Optimization design of magnetic valve controllable reactor," Guangdong Electric Power, vol. 26, pp. 7779, 2013.
[6] C. Hanxiang, H. Shaoyang, and H. Chaoxian, "Analysis of the electromagnetic characteristics of magnetically controlled reactor," Transformer, vol. 50, pp. 16-20, 2013.

[7] B. Chen, and J.M. Kokernak, "Thyristor controlled two-stage magnetic-valve reactor for dynamic var-compensation in electric railway power supply systems," In: Proceedings of the APCE, 2000, pp. 1066-1072.

[8] S. Jiangbao, W. Heping, and Z. Zhanyong, "The analysis of three-phase magnetism valve type controllable reactor," Power System Protection and Control, vol. 37, pp. 20-22, 2013.

[9] C. Xuxuan, C. Baichao, and T. Cuihua, "Two-stage saturable magnetically controlled reactor harmonic suppression optimization technique," Electric Power Automation Equipment, vol. 31, pp. 71-74, 2011.

[10] C. Xuxuan, T. Cuihua, and C. Baichao, "Mathematical model for harmonic analysis of multi-stage saturable magnetic valve controllable reactor", Journal of Electrician Technique, vol. 26, pp. 57-64, 2013.

Received: September 16, 2014

Revised: December 23, 2014

Accepted: December 31, 2014

(C) Xiangzheng Xu; Licensee Bentham Open

This is an open access article licensed under the terms of the Creative Commons Attribution Non-Commercial License (http://creativecommons.org/licenses/by-nc/4.0/) which permits unrestricted, non-commercial use, distribution and reproduction in any medium, provided the work is properly cited. 